\title{
Coliform Enteritis of Cottontail Rabbits
}

\author{
THOMAS M. YUILL AND ROBERT P. HANSON \\ Department of Veterinary Science, University of Wisconsin, Madison, Wisconsin
}

Received for publication 11 April 1964

\begin{abstract}
Yuill, Thomas M. (University of Wisconsin, Madison), and Robert P. Hanson. Coliform enteritis of cottontail rabbits. J. Bacteriol. 89:1-8. 1965.-Mortality from enteritis occurred among 78 penned adult cottontail rabbits (Sylvilagus floridanus) during severe winter weather, and in 9 juvenile rabbits held in captivity during the summer. This mortality was associated with high numbers of Escherichia coli throughout the intestinal tract. Grossly normal cottontails from the laboratory, from the outdoor penned colony, and from the wild had few $E$. coli, and these were usually restricted to the lower gut. It was possible to induce mortality of adult rabbits by holding them at $-20 \mathrm{C}$ for 2 to 14 days. The animals that died during exposure to low temperatures had signs of enteritis and high numbers of $E$. coli throughout the intestinal tract. It was possible to prolong the life of cottontails held at $-20 \mathrm{C}$ by antibiotic suppression of their intestinal $E$. coli. A marked slowing in the rate of passage of materials through the intestine ensued after exposure at $-20 \mathrm{C}$. This phenomenon may have been important in the alteration of the distribution of the intestinal microflora. Limited infection by $E$. coli occurs in rabbits in the wild. Of 47 serum samples from grossly normal cottontails collected in the wild, 42 contained $E$. coli agglutinins. The rabbit's defenses are apparently sufficient to control this organism under all but extremely unfavorable circumstances. The age of the rabbit appeared to influence its susceptibility to enteritis. Juvenile rabbits experienced enteritis, tissue invasion, and death under conditions which did not produce any enteric changes in adults.
\end{abstract}

This paper reports mortality of penned adult and juvenile cottontail rabbits (Sylvilagus floridanus), and attempted determination and experimental duplication of the causes of mortality.

Environmental conditions in winter may impose hardships upon cottontail rabbits in the wild. Deep snow and cold weather are reported to cause weight loss in wild cottontails (Allen, 1938; Haugen, 1942). Severe environmental conditions may make rabbits more susceptible to disease. Leopold (1931) reported observations of sick and dead cottontails in Wisconsin, Illinois, and Indiana during the winter of 1928-29. Winter in the more northern states has been described as a critical time for game populations (Allen, 1954).

In February, 1960, we released 136 wildtrapped cottontail rabbits from the city of Manitowoc, Wis., into outdoor pens at the State Conservation Department Game Farm at Poynette. One male and two female rabbits each were in pens 26 by $60 \mathrm{ft}$ in size, with one brush pile per pen for shelter. Alfalfa rabbit pellets were fed $a d$ libitum in trough-type covered feeders. Apple twigs were fed to the rabbits in late February and March. Rabbits began to die 7 to 10 days after release into the pens, and deaths continued through March. Most animals were found frozen in a normal crouched position behind sheltering posts, in the brush piles, and in snow forms. During this time, there were 17 nights when the temperature was $-25 \mathrm{C}$ or below. March was especially cold, averaging $14 \mathrm{C}$ below normal, and with a monthly mean of $-15 \mathrm{C}$. Necropsy of 13 of the 78 animals that died revealed circulatory congestion of the ileum and duodenum, and traces of frank blood in the lumen of these intestinal regions. Diarrhea was not observed. Without exception, the rabbits died with stomachs and intestinal tracts full of food. In 12 of 13 cases, deposits of intraperitoneal fat, which are nearly always present in cottontails during early winter, were absent.

During the following summer, 60 juvenile cottontails born in captivity and in the wild were reared by us in the laboratory. Eleven died not long after they were captured. Eight of these rabbits were removed from the nest at about 5 days of age and reared by hand. Deaths occurred 2 to 9 days later. In six of eight cases, petechial and ecchymotic hemorrhages of the ileum, duodenum, and caecum occurred, and there was frank blood in the lumen of the small intestine. The remaining three rabbits were older at the 
time of capture. One juvenile cottontail was trapped by a cooperator. The cooperator restrained the animal by securing its hind feet together with a rubber band, and placed the rabbit in a shoe box. When the animal was examined several hours later, it was weak and unable to stand. Pentamethylenetetrazole and glucose were administered, and the rabbit was placed in an incubator at $37 \mathrm{C}$. The rabbit appeared to have recovered $7 \mathrm{hr}$ later, but it died the following morning. Diarrhea and hemorrhaging into the lumen of the small intestine and caecum were noted at necropsy. Another juvenile cottontail had been in captivity for 3 days when it died. Postmortem examination revealed petechial hemorrhages of the caecum and distal end of the ileum. The final juvenile cottontail had been in captivity for 2 weeks when it died. At necropsy, petechial hemorrhages of the duodenum, jejunum, and proximal portions of the ileum were observed.

Descriptions of diseases of domestic animals which are associated with unfavorable environmental conditions and characterized by enteritis appear in the literature. Infectious diarrhea of newborn calves is precipitated by chilling and is caused by overgrowth of Escherichia coli, an organism of the animal's intestinal microflora (Reisinger, 1957). A similar disease of lambs was reported by Marsh and Tunnicliff (1938). In England, chickens shipped from distances of 200 miles had greater condemnation rates due to visceral lesions than did locally procured birds (Frey, personal communication).

\section{Materials and Methods}

Ten pools of spleen, liver, and kidney tissue from dead Game Farm adults were placed into screwcap vials and stored at $-20 \mathrm{C}$ until they were processed. After quick thawing, the tissues were ground with a TenBroeck tissue grinder, and a 10\% suspension was prepared with Tryptone (Difco) broth containing antibiotics (5,000 units of penicillin and $5 \mathrm{mg}$ of dihydrostreptomycin per $\mathrm{ml}$ ). Tissue suspensions were inoculated into 10-dayold embryonating white leghorn hen eggs $(0.2 \mathrm{ml}$ per egg into the allantoic chamber, 12 eggs per pooled sample), into 21-day-old weanling mice $(0.1 \mathrm{ml}$ intraperitoneally, five mice per pooled sample), and into juvenile cottontail rabbits (1.0 $\mathrm{ml}$ intraperitoneally, one rabbit per pooled sample). Tissue pools from dead juvenile rabbits were processed similarly, but they were not usually frozen prior to processing.

On the supposition that enteritis of bacterial or viral etiology - triggered by the stress of prolonged cold weather, moving, and temporary crowdingmay have been responsible for the mortality, we cultured contents of intestinal tracts and selected tissues for their microflora. Flora of the intestinal tract was determined by removing 6 inches of the duodenum, ileum, caecum, and colon, and stripping out the contents with sterile forceps immediately after thawing. A 1-g amount (wet weight) was secured from each of the four regions. After weighing, the sample was transferred to $100 \mathrm{ml}$ of sterile distilled water and homogenized by shaking with glass beads. Pour plates of eosin-methylene blue agar (EMB, Difco), MacConkey Agar (Difco), and lactobacillus selective agar (LBS, BBL) were made with $10^{-3}, 10^{-4}$, and $10^{-5}$ dilutions of intestinal contents. Thick Thioglycollate (Difco) plates were poured with dilutions of $10^{-4}$, $10^{-5}$, and $10^{-6}$. EMB, MacConkey, and Thioglycollate plates were incubated aerobically, and LBS plates were incubated in an atmosphere containing $10 \% \mathrm{CO}_{2}$. All cultures were incubated for $48 \mathrm{hr}$ at $37 \mathrm{C}$. The rapid replica plating technique of Wiseman and Sarles (1955) was employed for the identification of coliforms from Thioglycollate cultures. Organisms presenting typical $\boldsymbol{E}$. coli characteristics on EMB agar were subjected to the IMViC test (Burrows, 1959).

During postmortem examinations of the juvenile rabbits, material from liver, kidney, and heart blood was streaked on EMB, Albimi brucella agar (Albimi Laboratories, Flushing, N.Y.), Blood Agar Base (Difco) containing 5\% sheep blood, and MacConkey Agar.

\section{RESUlTS}

Suspended tissues from dead adult and juvenile rabbits gave no indication of the presence of a viral agent when inoculated into mice, eggs, and cottontail rabbits.

No Salmonella or Shigella cells were isolated. $E$. coli was present in all animals and more widely distributed through the intestinal tract than would be expected in normal animals (Fig. 1). All adult rabbits had $E$. coli counts of $10^{3}$ to more than $10^{8}$ per 1 -g sample of intestinal contents. Samples from all of the ten adults proc-

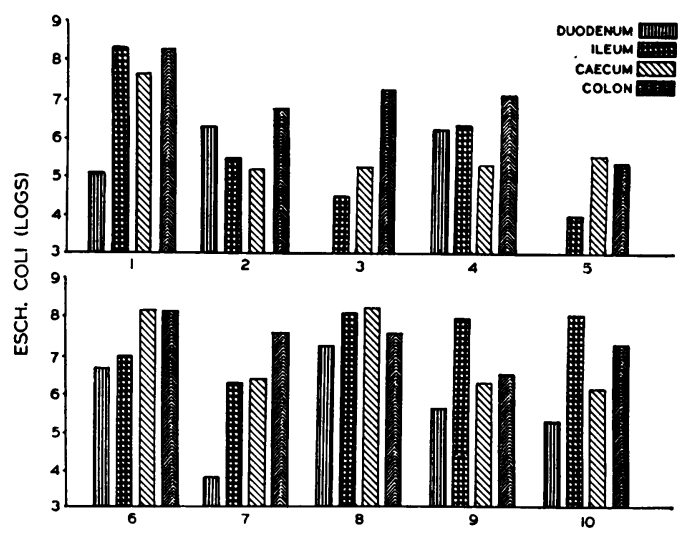

FIg. 1. Numbers of intestinal Escherichia coli from cottontail rabbits dying spontaneously in an outdoor colony during cold weather. 
essed had $E$. coli present in numbers exceeding $10^{3}$ in caecum, colon, and ileum. Eight of these rabbits had this number of $E$. coli in the duodenum (Fig. 1). No species of Lactobacillus was detected, even when the cultures were incubated an additional $48 \mathrm{hr}$. Other bacteria, including Paracolobactrum spp., Proteus sp., and Aerobacter sp., were found, but were considerably less abundant than $E$. coli.

No attempt was made to isolate bacteria from the tissues of adult rabbits. $E$. coli was isolated from heart blood, liver, and kidney cultures of 9 of 11 dead juvenile rabbits. These nine individuals were those that showed hemorrhagic enteritis.

We postulated that under unfavorable environmental conditions, $E$. coli was able to multiply and cause the death of adult rabbits. Experiments were carried out to test this hypothesis. Unless otherwise stated, adult cottontails used in these experiments were live-trapped on the University of Wisconsin Arboretum and were held in captivity no longer than 5 days prior to experimental use.

For comparison with numbers observed in the diseased cottontails, we enumerated $E$. coli in the intestines of normal cottontail rabbits. During the first week of March, 1960, while mortality was still occurring at the State Game Farm, four of the surviving and apparently normal rabbits were taken to the laboratory. Two of these animals were killed, and samples of the intestinal contents were taken immediately. These rabbits harbored low numbers of $E$. coli $\left(10^{5}\right.$ organisms per $1 \mathrm{~g}$ of contents) in the lower intestine only. To test the possibility of extensive postmortem multiplication of $E$. coli, the remaining two animals were killed, and the intact carcasses placed at $-20 \mathrm{C}$. It took slightly under $8 \mathrm{hr}$ for these animals to freeze solidly. The frozen rabbits were then thawed and processed in exactly the same manner as those animals experiencing mortality in the outdoor pens. These rabbits had no detectable numbers of $E$. coli at dilutions of $10^{-3}$ or over. Two cottontails from the laboratory colony (one had been in captivity for 3 months, the other for 9 months) were killed, and pour plates were made of dilutions of intestinal contents. No intestinal $E$. coli cells were detected in dilutions of $10^{-3}$ or greater.

As a further source of information on numbers of intestinal coliforms, three cottontails were trapped in late December, 1960, in the University of Wisconsin Arboretum, killed by the trapper, and transported to the laboratory. When intestinal contents were taken, we observed that one rabbit was freshly dead (still warm), and the other two were cold and had gas accumulat- ing in the caecum. The trapper later reported that two of the three rabbits had been killed in the early morning. The rabbits had been transported in the heated cab of a vehicle until delivery to the laboratory. We strongly suspect that during the several hours they were in the warm truck, postmortem multiplication of $E$. coli had occurred in the two rabbits which had been dead the longest. The rabbit most recently dead had no measurable numbers of $E$. coli at dilutions at $10^{-3}$ and above. During the following February, three cottontails were shot on the Arboretum. Intestinal tracts were immediately removed, placed in plastic freezer bags, and cooled in the snow. They were then transported to the laboratory in an unheated vehicle, frozen at $-20 \mathrm{C}$ for 2 days, and then processed in the usual manner. None of these rabbits had measurable levels of $E$. coli at dilutions of $10^{-3}$ or above.

We determined some effects of exposure of cottontail rabbits to large quantities of $E$. coli organisms and endotoxin. The strain of $E$. coli TMY used originated from a caecal isolate from an apparently grossly normal cottontail. The culture has been supplied to CDC for typing. Shake cultures $(24 \mathrm{hr}$ ) were grown in Tryptone broth at $37 \mathrm{C}$ and washed twice in physiological saline before administration.

A 7-ml amount of a stock suspension of viable $E$. coli titering $10^{9}$ organisms per milliliter was administered to three cottontails, $5 \mathrm{ml}$ via stomach tube and $2 \mathrm{ml}$ into the colon. Although no deaths occurred, intestinal $E$. coli counts at $24 \mathrm{hr}$ (Fig. 2) were higher than normal controls.

Four cottontails were given $7 \mathrm{ml}$ of viable organisms titering $10^{9}$ per $\mathrm{ml}$ intraperitoneally. One rabbit died in $5 \mathrm{hr}$, and another died sometime between 6 and $15 \mathrm{hr}$. In the former rabbit, intestinal contents contained high numbers of $E$. coli throughout, and this organism was also isolated from heart blood, spleen, and liver. As the latter rabbit had been dead for several hours when discovered, neither tissues nor intestinal contents were cultured. The two surviving rabbits harbored low numbers of $E$. coli in their small intestines.

Endotoxin can be liberated by ultrasonic vibration. A live culture of $E$. coli, which titered $10^{11}$ organisms per milliliter, received $45 \mathrm{~min}$ of ultrasonic vibration in a Raytheon ultrasonic oscillator. This treatment produced over $99.9 \%$ mortality $\left(10^{6}\right.$ viable organisms per milliliter remaining). The sonically treated culture was then passed through a Seitz EK filter, and bacterial sterility was confirmed prior to use. This endotoxin preparation had an intracerebral LD $_{50}$ of 1:32 in 3-day-old suckling mice. Three cottontails were given $7 \mathrm{ml}$ of the filtered endo- 

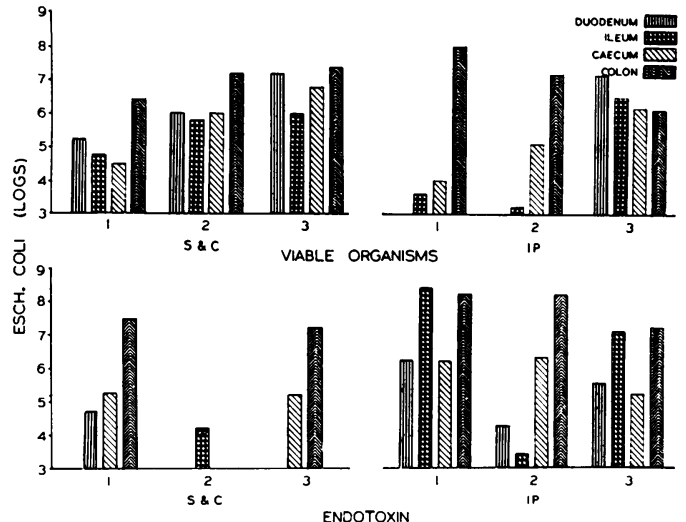

Fig. 2. Numbers of intestinal Escherichia coli from cottontail rabbits given endotoxin and viable organisms into the stomach and colon $(S \& C)$ and intraperitoneally $(I P)$.

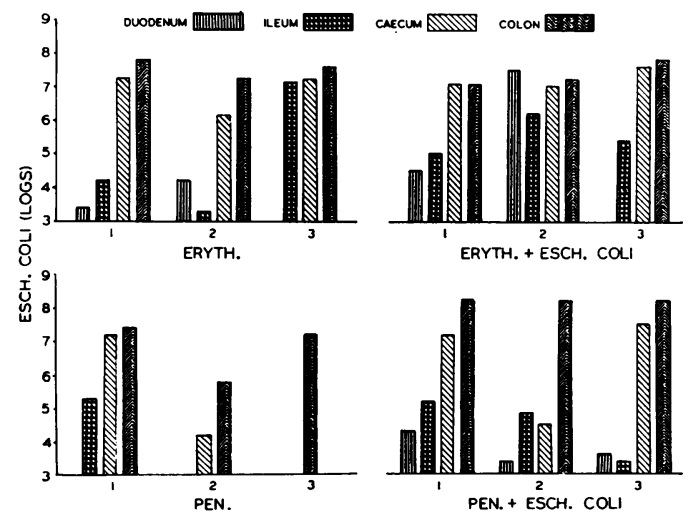

FIG. 3. Numbers of intestinal Escherichia coli from cottontail rabbits after administration of erythromycin (eryth.) and penicillin (pen.). Half the rabbits were given $E$. coli into the stomach and colon, $48 \mathrm{hr}$ after administration of the drugs.

toxin, $5 \mathrm{ml}$ by stomach tube and $2 \mathrm{ml}$ into the colon. No deaths occurred, nor were $E$. coli numbers raised significantly in the intestinal tract after $24 \mathrm{hr}$.

Three cottontails received $7 \mathrm{ml}$ of endotoxin intraperitoneally. One rabbit died in $18 \mathrm{hr}$, and high numbers of $E$. coli were found throughout the intestinal tract. Severe hemorrhagic enteritis was observed, and $E$. coli was isolated from the heart blood, liver, and spleen. One live rabbit, killed $24 \mathrm{hr}$ after inoculation, also showed signs of hemorrhagic enteritis, and isolations of $E$. coli were made from the heart blood, liver, and spleen. Titrations of intestinal-content samples revealed high numbers of $E$. coli throughout the tract. The third rabbit was grossly normal when killed $24 \mathrm{hr}$ after inoculation. Numbers of $E$. coli in the intestinal tract were low, and no isolations were made from other tissues (Fig. 2).

Cortisone has been used to suppress antibody formation in domestic rabbits (Germuth and Ottimyer, 1950; Mogabgab and Thomas, 1952; Robinson and Smith, 1953). We postulated that cortisone might produce stress and enhance susceptibility, permitting $E$. coli to multiply and to kill the host. Cortisone was administered intramuscularly to six cottontails at the rate of $5 \mathrm{mg}$ per day for 4 days. At $24 \mathrm{hr}$ after the last injection, three of the rabbits were given $7 \mathrm{ml}$ of the stock $E$. coli suspension (titering $10^{11}$ viable organisms per milliliter), $5 \mathrm{ml}$ via stomach tube and $2 \mathrm{ml}$ into the colon. At $48 \mathrm{hr}$ after inoculation, all six cottontails were killed, and 1-g samples of contents were taken from the usual four intestinal regions. An additional group of three cottontails was given $15 \mathrm{mg}$ of cortisone acetate intramuscularly for 7 days, killed on day nine, and samples of intestinal contents were plated out in the usual fashion. There was no marked increase in the numbers of $E$. coli.

In work with guinea pigs, De Somer et al. (1955) produced $90 \%$ mortality by intraperitoneal administration of 100,000 units of penicillin. Death occurred in $48 \mathrm{hr}$ owing to reduction of normal gram-positive flora with a resultant overgrowth of $E$. coli throughout the intestinal tract.

Six cottontails were each given 500,000 units of crystalline penicillin $\mathrm{G}$ (reconstituted in physiological saline) intraperitoneally. After 48 hr, three rabbits were given viable $E$. coli, titering $10^{11}$ organisms per milliliter, which was administered into the colon and stomach. These animals were killed $48 \mathrm{hr}$ later, and $E$. coli from samples of intestinal contents were enumerated in the usual manner. In only one rabbit was the $E$. coii count raised to particularly high levels in the small intestine (Fig. 3). In this individual, no pathological signs were observed, nor were organisms isolated from the spleen, liver, or heart blood.

Drug-sensitivity tests indicated that in vitro growth of the stock strain of $E$. coli was not inhibited by erythromycin. Because erythromycin is excreted into the intestinal tract by the liver and is effective against gram-positive organisms (Jones, 1960), it was used in an attempt to produce overgrowth of $E$. coli. Six cottontails were each given $50 \mathrm{mg}$ of erythromycin (Erythrocin, Abbott Laboratories, North Chicago, Ill.), intramuscularly. At $48 \mathrm{hr}$ after injection, the stock suspension of $E$. coli was administered in the usual quantities into the 
stomach and colon. All animals were killed 48 $\mathrm{hr}$ after administration of organisms, and samples of the intestinal contents were plated out in the usual fashion. In two of the animals, $E$. coli counts were elevated in both the large and small intestines. No pathological effects were noted, nor were any organisms isolated from the heart blood, liver, or spleen.

We attempted to duplicate conditions under which mortality in the pens occurred. Six cottontails were individually caged and placed in a walk-in freezer at $-20 \mathrm{C}$. They were fed shelled corn ad libitum. A supply of water, in the form of a small block of ice, was always available in each cage. Three of the rabbits were given $7 \mathrm{ml}$ of the stock culture $E$. coli ( $10^{11}$ organisms per milliliter), $5 \mathrm{ml}$ via stomach tube and $2 \mathrm{ml}$ into the colon. Three controls were given similar treatment with sterile saline substituted for the suspension of organisms. Five of the six cottontails died between days 5 and 14. The sixth cottontail was killed on day 14 . Both groups died at the same rate. All animals had high numbers of $E$. coli throughout the intestinal tract (Fig. 4). In all rabbits, enlarged mesenteric lymph nodes and congestion of the small intestine were noted at necropsy. All of the five rabbits that died exhibited signs of enteritis. Four animals had petechial hemorrhages of the mucosa, and frank blood in the lumen of much of the small intestine; the fifth rabbit had adhesions of the duodenum and upper jejunum to other regions of the intestinal tract and to the body wall. The most severe enteritis was noted among those animals given live $E$. coli. This appeared to be the same condition that was present in spontaneously occurring mortality among the adult rabbits from the State Game Farm. E. coli was not isolated from the spleen, liver, or heart blood.

With antibiotics, we attempted to prevent extensive intestinal multiplication of $E$. coli in cottontails held at $-20 \mathrm{C}$. The stock $E$. coli strain was sensitive to oxytetracycline (Terramycin, Chas. Pfizer \& Co., Inc., Brooklyn, N.Y.). Eight rabbits were held at $-20 \mathrm{C}$ and maintained on a diet of shelled corn. Four cottontails each received $50 \mathrm{mg}$ of oxytetracycline via stomach tube in the morning and evening, and had an additional $100 \mathrm{mg}$ mixed with their corn. Four controls received an equal volume of saline via stomach tube. This experiment was conducted in mid-March at a time when the rabbits had begun their spring molt. This factor is believed to account for the shortened life of this group as compared to the previous coldtemperature experimental group tested a month earlier. The oxytetracycline-treated group lived

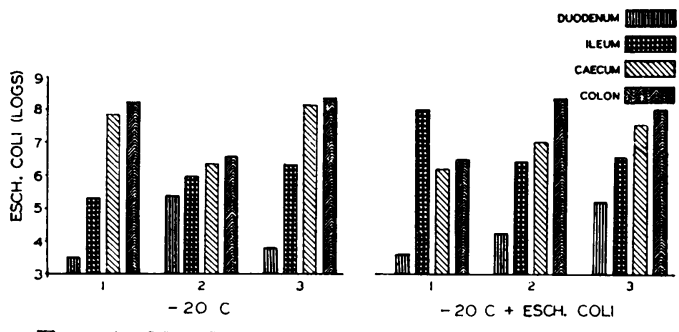

FIG. 4. Numbers of intestinal Escherichia coli from cottontail rabbits kept at $-20 \mathrm{C}$, with and without administration of $E$. coli into the colon and stomach.

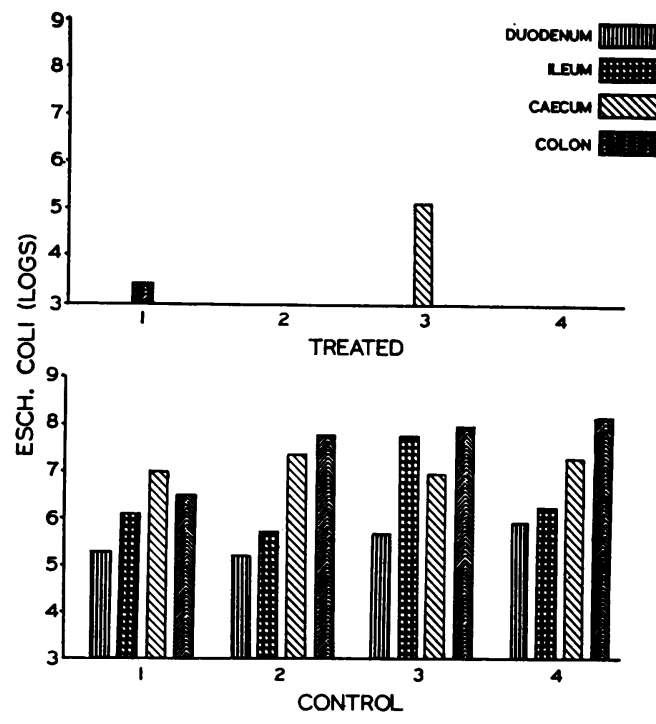

Fig. 5. Effect of oxytetracyline treatment on numbers of intestinal Escherichia coli from cottontail rabbits kept at $-20 \mathrm{C}$.

an average of 10.0 days and had no measurable numbers of $E$. coli in samples of intestinal contents in dilutions of $10^{-3}$ or greater (Fig. 5). No pathological changes suggestive of enteritis were noted at necropsy, and the animals were presumed to have succumbed to the prolonged exposure to low temperatures. The untreated animals did have measurable numbers of $E$. coli in the upper intestinal tract (Fig. 5) and lived an average of only 3.5 days. At necropsy, enlarged mesenteric lymph nodes, petechial hemorrhaging into the lumen of the terminal end of the ileum, and congestion of the small intestine occurred in all four untreated animals.

Theorizing that motility of the gut was an important aspect in the distribution of microflora, we measured the effect of low environmental temperatures on the rate of passage of 
materials through the gastrointestinal tract. Seven cottontail rabbits were given $10 \mathrm{ml}$ of a suspension of powdered calcium carbonate via stomach tube. Four of these animals were held at room temperature $(24 \mathrm{C})$. The remaining three rabbits were kept at $-20 \mathrm{C}$ for $48 \mathrm{hr}$ prior to administration of calcium carbonate. The dropping pans under the cages were examined every $0.5 \mathrm{hr}$ for the appearance of white feces. Rabbits held at room temperature had a mean rate of passage of $8.8 \mathrm{hr}$ (ranging from 9 to 10.5 $\mathrm{hr}$ ). Those held at $-20 \mathrm{C}$ had a mean rate of passage of $15.5 \mathrm{hr}$ (ranging from 14.5 to $17 \mathrm{hr}$ ).

Prolonged exposure to low temperatures may have damaged the integrity of the gut and produced increased uptake of $E$. coli endotoxins. Experiments to demonstrate damage were inconclusive.

To determine whether or not $E$. coli became invasive in nature, we determined the frequency of $E$. coli $\mathrm{K}$ agglutinins from a sample of normal adult wild rabbits. We measured titers of $E$. coli $\mathrm{K}$ and $\mathrm{O}$ agglutinins in 24 individuals collected from 15 December 1959 through 24 February 1960 and from 23 individuals taken during the same period 1 year later. For bacterial tube agglutinations, we used whole blood eluted from absorbent paper discs, and sera. Doubling dilutions were made starting with a $1: 10$ dilution of sera or a 1:5 dilution of whole blood eluate. The agglutinogen consisted of the stock culture of $E$. coli diluted in physiological saline to $50 \%$ transmissibility at a wavelength of $612 \mu$, by use of a Coleman Junior spectrophotometer. The agglutination tubes were incubated at $45 \mathrm{C}$ for $1 \mathrm{hr}$, and then at room temperature overnight.

Agglutinating titers of $1: 160$, or higher, to $E$. coli were present in 12 individuals, and in only 5 of the 47 sera tested were no antibodies detected. The mean agglutinin titers from rabbits collected during the severely cold winter of 1959-60 was $1: 188$; this was not substantially higher than the mean agglutinin titer of $1: 117$ from animals collected during the mild winter of 1960-61. Because of the likelihood of strain variation among individuals, more animals may have had $E$. coli agglutinins than we detected with an agglutinogen from a single rabbit.

\section{Discussion}

$E$. coli in the intestinal tract normally maintains a commensal relationship with its host. Under some circumstances, $E$. coli becomes pathogenic. Certain serotypes of this organism are the etiological agents responsible for some enteric infections of man (Greenberg and
Wronker, 1938), lambs (Marsh and Tunnicliff, 1938; Roberts, 1957, 1958), calves (Reisinger, 1957), dogs (Mansi, 1961), and poultry (Gross, 1961), and are strongly implicated in swine (Sojka, Erskine, and Lloyd, 1957; Timoney, 1957). Sheep (Marsh and Tunnicliff, 1938) and calves (Smith and Orcutt, 1925; Reisinger, 1957) are reported to be predisposed to enteric infection by $E$. coli by chilling. Roberts (1957, 1958) reported that physical stress (unspecified) was an important factor in enteritis of $E$. coli etiology in Australian lambs. Newcastle disease vaccination predisposed chickens to pericarditis of $E$. coli origin (Gross, 1961). Host age also influences the pathogenesis of $E$. coli enteritis. Enteritis was reported in lambs (Marsh and Tunnicliff, 1938; Roberts, 1957, 1958) and calves (Reisinger, 1957), but not in mature animals. Human volunteers fed $E$. coli, isolated from infants with severe gastroenteritis, developed a milder disease (Ferguson and June, 1952). Mansi (1961) reported that gastroenteritis caused by $E$. coli produced fewer fatalities in adult dogs than in younger dogs.

Changes in gastrointestinal microflora can be rapid. Jacob et al. (1954) reported that dogs experiencing experimentally induced hemorrhagic shock often died within a few hours owing to toxemia and tissue invasion of rapidly multiplying $E$. coli and clostridia. When these workers therapeutically reduced the numbers of $E$. coli and clostridia in the intestines of the dogs prior to hemorrhagic shock, mortality was reduced from 80 to $20 \%$.

In adult cottontail rabbits, we observed that the presence of high numbers of $E$. coli throughout the intestine was associated with mortality only when the animals were exposed to low temperatures. Experimentally, most of the cottontails developed enteritis and died after 2 to 14 days at $-20 \mathrm{C}$. Large numbers of intestinal $E$. coli, induced by antibiotics or per os administration of viable organisms, did not affect adult cottontails kept at room temperature. When $E$. coli was suppressed by oral administration of oxytetracycline, treated animals lived an average of 6.5 days longer at $-20 \mathrm{C}$ than did untreated animals, and exhibited no signs of enteritis at death (untreated animals did). This observation is consistent with the concept that $E$. coli was capable of becoming invasive in rabbits and causing mortality when the rabbits were exposed to low temperatures. The fact that $89 \%$ of the sera collected from grossly normal wild cottontails contained $E$. coli agglutinins indicates that, in nature, this organism may frequently cause infections. The 
rabbit's bodily defenses are apparently able to cope with the infection under favorable environmental circumstances.

Cold has been reported to decrease the frequency of peristaltic contractions of the intestine in the dog (Gladkova, 1959). We did not determine if the slowing of the rate of passage of materials through the intestinal tract in rabbits held at $-20 \mathrm{C}$ preceded overgrowth by $E$. coli. Loss of gut motility prior to extensive multiplication of $E$. coli might have permitted the rapid spread of $E$. coli into the small intestine and the development of a high $E$. coli population throughout the intestine. Loss of motility of the gut after overgrowth by $E$. coli would have produced a longer period during which endotoxins could have been absorbed.

It does not seem likely that high numbers of $E$. coli throughout the intestinal tract occurred only in animals which were moribund from exposure to cold. One rabbit given sterile $E$. coli endotoxin intraperitoneally was not yet moribund, but had begun to sustain gross damage to the mucosa of the small intestine and to harbor high numbers of $E$. coli throughout the intestinal tract. Conversely, rabbits killed and placed at $-20 \mathrm{C}$ had no measurable multiplication of $E$. coli in their intestinal tracts, even though 8 $\mathrm{hr}$ were required for the carcasses to freeze solidly.

The age of the host appeared to play a role in the susceptibility to and development of terminal effects of $E$. coli enteritis. Juvenile rabbits experienced intestinal $E$. coli overgrowth, enteritis, and death under conditions in the laboratory which produced no enteric disturbance in adults. Tissue invasion by $E$. coli occurred in juvenile rabbits but not in adults (except those inoculated intraperitoneally). In a review of the role of $E$. coli in disease, Krammer (1961) stated that age is a very important factor in susceptibility of the host to $E$. coli infections.

Multiplication and spread of $E$. coli throughout the intestine, combined with favorable environmental conditions, apparently were responsible for the mortality in penned adult and juvenile rabbits. In the laboratory and in the field, exposure of adult cottontails to low temperatures was an important factor in precipitating $E$. coli overgrowth. In juvenile cottontails, captivity induced $E$. coli enteritis. Quantitative data, such as determination of the effects of high population densities on rabbits and the measurement of microclimatic conditions prevailing in the rabbit's environment, are needed before the significance of $E$. coli enteritis can be extrapolated to populations in the wild.

\section{Acknowledgment}

The senior author carried out this work during the tenure of a Wisconsin Alumni Research Foundation Fellowship.

\section{Literature Cited}

Allen, D. L. 1938. Breeding of the cottontail rabbit in southern Michigan. Amer. Midland Naturalist 20:464-469.

Allen, D. L. 1954. Our wildlife legacy, p. 46-48. Funk and Wagnalls Co., New York.

Burrows, W. 1959. Textbook of microbiology, p. 461. W. B. Saunders Co., Philadelphia.

DeSomer, W. W., H. Van De Voorde, H. Essen, AND P. VAN DiJCK. 1955. A study on penicillin toxicity in guinea pigs. Antibiot. Chemotherapy 5:463-468.

Ferguson, W. W., and R. C. June. 1952. Experiments on feeding adult volunteers with Escherichia coli III B, a coliform. Amer. J. Hyg. 55: 155-169.

Germuth, R. G., and B. Ottimyer. 1950. Effect of 17 hydroxy 11-dehydrocorticosterone (compound $\mathrm{E}$ ) and of ACTH on arthus reaction and antibody formation in the rabbit. Proc. Soc. Exp. Biol. Med. 74:815-823.

Gladkova, A. I. 1959. Thermoinfluences and motor activity of the intestine. Sechenov Physiol. J. USSR (English Transl.) 45:84-91.

Greenberg, M., ANd B. M. Wronker. 1938. An outbreak of epidemic diarrhea in the newborn. J. Amer. Med. Assoc. 110:563-566.

Gross, W. B. 1961. Escherichia coli as a complicating factor in Newcastle disease vaccination. Avian Diseases 5:132-134.

HAUGEN, A. O. 1942. Life history studies of the cottontail rabbit in southwestern Michigan. Amer. Midland Naturalist 28:204-244.

JaCOB, S., H. Wizel, E. Gordon, H. Korman, F. Schweinberg, H. Frank, and J. J. Fine. 1954. Bacterial action in development of irreversibility to transfusion in hemorrhagic shock in the dog. Amer. J. Physiol. 179:523-531.

Jones, L. M. 1960. Veterinary pharmacology and therapeutics, p. 459-461. Iowa State College Press, Ames.

Kramer, T. 1961. The problems of pathogenicity of Escherichia coli in animals and man. Can. Vet. J. 2:326-328.

LEOPOLD, A. 1931. Report on a game survey of the north central states, p. 93-94. Sporting Arms and Ammunition Manufacturers Institute, Madison.

MaNSI, W. 1961. Is E. coli infection of significance in the dog? Mod. Vet. Pract. 42:41-42.

Marsh, H., and E. A. Tunnicliff. 1938. Dysentery of newborn lambs. Montana State Coll. Agr. Exp. Sta. Bull. 361.

Mogabgab, W. J., and L. Thomas. 1952. Effects of cortisone on bacterial infection. J. Lab. Clin. Med. 39:271-289.

Reisinger, R. 1957. Studies on the pathogenesis of infectious diarrhea in newborn calves. M.S. Thesis, University of Wisconsin, Madison. 
Roberts, D. S. 1957. Escherichia coli infection in lambs. Australian Vet. J. 33:43-45.

RoberTs, D. S. 1958. Further observations on $E$. coli disease in lambs. Australian Vet. J. 34:152156.

Robinson, H. J., AND A. J. Smith. 1953. Effect of adrenal cortical hormones on experimental infection. Ann. N.Y. Acad. Sci. 56:757-764.

Smith, T., AND M. L. ORCUTt. 1925. Bacteriology of the intestinal tract of young calves with spe- cial reference to the early diarrhea (scours). J. Exp. Med. 41:81-106.

SoJKA, W. J., R. J. Erskine, and M. K. Lloyd. 1957. Haemolytic Escherichia coli and "oedema disease" of pigs. Vet. Rec. 69:293-301.

Timoney, J. F. 1957. Oedema disease in swine. Vet. Rec. 69:1160-1171.

Wiseman, R. F., aND W. B. Sarles. 1955. A plating technique for screening intestinal coliform bacteria. J. Bacteriol. 71:480-481. 